Shakesplish 


\section{SQUARE ONE}

First Order Questions in the Humanities

PAUL A. KOTTMAN, EDITOR 


\section{SHAKESPLISH}

How We Read Shakespeare's Language

\section{Paula Blank}


Stanford University Press

Stanford, California

(C) 2018 by the Board of Trustees of the Leland Stanford Junior University. All rights reserved.

No part of this book may be reproduced or transmitted in any form or by any means, electronic or mechanical, including photocopying and recording, or in any information storage or retrieval system without the prior written permission of Stanford University Press.

Printed in the United States of America on acid-free, archival-quality paper

Library of Congress Cataloging-in-Publication Data

Names: Blank, Paula, 1959-2016, author.

Title: Shakesplish : how we read Shakespeare's language / Paula Blank.

Description: Stanford, California : Stanford University Press, 2018. | Series: Square one : firstorder questions in the humanities | Includes bibliographical references and index.

Identifiers: LCCN 2018019692 (print) | LCCN 2018021762 (ebook) | ISBN 9781503607583 | ISBN 9780804791939 (cloth : alk. paper) | ISBN 9781503607576 (pbk. : alk. paper)

Subjects: LCSH: Shakespeare, William, 1564-1616-Language. | Shakespeare, William, 15641616-Literary style. | English language-Early modern, 1500-1700. | English languagePsychological aspects.

Classification: LCC PR3072 (ebook) | LCC PR3072 .B53 2018 (print) | DDC 822.3/3-dc23

LC record available at https://lccn.loc.gov/2018019692

Cover design: Christian Fuenfhausen 